\title{
TWO-DIMENSIONAL SPATIAL \\ POWER SPECTRA OF PHOTOSPHERIC VELOCITY \\ FLUCTUATIONS
}

RICHARD G. TESKE*

San Fernando Observatory, the Aerospace Corporation, Calif., U.S.A.

(Received 3 July; in revised form 23 September, 1974)

\begin{abstract}
Two-dimensional spatial autocorrelation functions and power spectral density distributions were obtained from high-resolution velocity spectroheliograms. Although the autocorrelation functions indicate the existence of velocity cells of size roughly 2500 to $3500 \mathrm{~km}$, the power spectra fail to reveal them because the cells are not strictly spatially periodic.
\end{abstract}

\section{Introduction}

Numerous direct observations of the solar photospheric oscillations have suggested that they exist with a fairly well-defined cell size of a few thousand $\mathrm{km}$. On the other hand, power spectrum analyses have sometimes failed to show a significant power peak at the expected wavenumber, especially in recent investigations. Wolf (1973) has reviewed the matter and has offered an interpretation.

A summary of published material is listed in Table I, where the results of various investigations have been converted to the uniform system of velocity cell 'diameter'.

TABLE I

Dimensions of velocity cells in solar photosphere

\begin{tabular}{|c|c|c|c|}
\hline \multicolumn{2}{|c|}{$\begin{array}{l}\text { Obtained from observations } \\
\text { cell size }(\mathrm{km})\end{array}$} & \multicolumn{2}{|c|}{$\begin{array}{l}\text { Obtained from power spectra } \\
\text { cell size }(\mathrm{km})\end{array}$} \\
\hline $1700-3500$ & Leighton et al. (1962) & $\sim 5000$ & Mein (1966) \\
\hline $2000-3000$ & $\begin{array}{l}\text { Evans and Michard } \\
(1962)\end{array}$ & $\sim 2500$ & Frazier (1968) \\
\hline $7000-10000$ & Roddier (1966) & $30000-40000$ & $\begin{array}{l}\text { Apenzeller and Schröter } \\
\text { (1968) }\end{array}$ \\
\hline 20004000 & Deubner (1967) & $\sim 10000$ & Tannenbaum et al. (1969) \\
\hline $5000-9000$ & Deubner (1969) & $\sim 3000$ & Beckers and Parnell (1969) \\
\hline \multirow[t]{2}{*}{$\sim 3000$} & Sheeley and Bhatnagar & & \\
\hline & $\begin{array}{l}\text { (1971) } \\
\text { Reif and Musman. }\end{array}$ & $\sim 3000$ & Mehltretter (1971) \\
\hline \multirow{3}{*}{$\sim 4300$} & $(1971)$ & $\sim 5000$ & Reiling (1971) \\
\hline & & $11000-18000$ & Deubner (1972) \\
\hline & & $5000-25000$ & $\begin{array}{l}\text { Edmonds and Webb } \\
\text { (1972b) }\end{array}$ \\
\hline
\end{tabular}

* Permanent address: University of Michigan, Ann Arbor, Mich. 48104, U.S.A. 
Direct observations tend to yield cell sizes in the range 2000 to $6000 \mathrm{~km}$. While the power spectrum results are sometimes in agreement with this range, disagreement also exists. Among those power spectrum analyses which do agree with the direct observations, some words of caution may be needed: for example, although Beckers and Parnell (1969) found a peak near $\log k \sim-3.0$ (cell size $3000 \mathrm{~km}$ ), it was not a strong one. Further, Frazier's (1968) analysis was based upon a limited spatial sample which produced poor resolution at spatial frequencies below the peak seen by him.

A possible explanation of the failure of some of the power spectra to reveal the oscillatory cells with certainty lies in the persistent use of one-dimensional data by the observers. At issue is the attempt to characterize from observations the horizontal wavenumber $k_{h}=\left(k_{x}^{2}+k_{y}^{2}\right)^{1 / 2}$ of a three-dimensional structure in the solar atmosphere. The question is whether power spectral densities, computed from one-dimensional data scans which cross randomly-oriented non-circular velocity structures at arbitrary places, have failed to portray the osciliating elements for this reason. In the present work, two-dimensional (spatial) power spectra have been constructed in an attempt to answer the question.

Leighton et al. (1962) claimed that vertical motions in the photosphere were dominated by the oscillating elements. Work by others (e.g., Deubner, 1969) has also tended to show the dominance of oscillatory motions. Thus single velocity 'snapshots' such as those used here are expected to be heavily weighted by the five-minute oscillations and to portray their spatial characteristics. The two-dimensional power spectral density distributions derived here fail to show clear evidence for a power peak at the expected wavelength of the 'classical' size of oscillatory elements. The failure is ascribable to the fact that velocity cells are not strictly spatially periodic.

\section{Observations}

Velocity spectroheliograms were made at the San Fernando Observatory in the wings of a $\mathrm{Ca}$ I $\lambda 6103$, using an image scale of $60 \mu \mathrm{m}$ per arcsec on the Sun. The instrument has been described by Mayfield (1971). An entrance slit width of $1.0^{\prime \prime}$ was used. The two exit slits, of width $60 \mathrm{~m} \AA$ in the spectrograph focal plane, were centered $60 \mathrm{~m} \AA$ from the core of the line. The solar image was scanned once per spectroheliogram at a rate of about 5.4" per second of time. Two pairs of spectroheliograms obtained during superior and uniform seeing conditions were selected for analysis. On them, photospheric structures of size approximately one arcsec or smaller were visible. Grain size of the SO 392 emulsion is about $5 \mu \mathrm{m}$, corresponding to about $0.1^{\prime \prime}$ on the Sun.

The individual spectroheliograms from the red and violet wings of the line were digitized on an Optronics P-1000 high-speed film reader using an $0.42^{\prime \prime}$ scanning aperture $\left(\lambda_{\text {Nyquist }}=612 \mathrm{~km}\right.$ on the Sun), and subtracted to provide a velocity picture in a computer. Various small portions of the two velocity pictures on the quiet photosphere were subjected to the analyses described below. 


\section{Results}

\subsection{VeLOCITY AND BRIGHTNESS FLUCTUATIONS}

The Doppler signal, brightness signal and brightness-velocity correlation coefficient were obtained in the manner described by Leighton et al. (1962). Let the Doppler signal be $\delta$ and let the brightness signal be $\beta$, both expressed as fractions of the mean intensity averaged over the picture. The digitized value of the photographic density at some position $(x, y)$ on a single scene made in the red or violet wing is $\chi(x, y)$. We may define a lagged mean square product when scene 2 is offset by $(s, t)$ relative to scene 1 :

$$
\sigma_{12}^{2}=\frac{1}{N} \sum\left\{\left[\chi_{1}(x, y)-\bar{\chi}_{1}\right]\left[\chi_{2}(x+s, y+t)-\bar{\chi}_{2}\right]\right\},
$$

where $N$ is the number of data points which overlap.

It may then be shown that (cf. Leighton et al., 1962) for $s=t=0$,

$$
\begin{aligned}
& \text { mean square brightness fluctuation }=\left\langle\beta^{2}\right\rangle=f_{1}\left(\sigma_{r r}^{2}, \sigma_{v v}^{2}, \sigma_{r v}^{2}, a\right) ; \\
& \text { mean square Doppler fluctuation }=\left\langle\delta_{v}^{2}\right\rangle=f_{2}\left(\sigma_{r r}^{2}, \sigma_{v v}^{2}, \sigma_{r v}^{2}, a\right) ; \\
& \text { brightness-velocity correlation coefficient }=C_{\beta \delta_{v}}=f_{3}\left(\sigma_{r r}^{2}, \sigma_{v v}^{2}, \sigma_{r v}^{2}, a\right) ;
\end{aligned}
$$

where $r r$ and $v v$ indicate autocorrelations for red wing and violet wing scenes and $r v$ designated the cross-correlation. The quantity $a$ takes account of the asymmetry of the $\mathrm{Ca} I \lambda 6103$ line and is defined by $\delta_{r}=a \delta_{v}$. The rms velocity fluctuation $\left\langle v^{2}\right\rangle^{1 / 2}$ may be obtained from $\left\langle\delta_{v}^{2}\right\rangle^{1 / 2}$ in the usual manner (Leighton et al., 1962). Calibrations and computational details are discussed by Teske (1974a).

Table II compares the values obtained from the San Fernando spectroheliograms

TABLE II

rms velocity and brigthness fluctuations and brightness-velocity correlation coefficients

\begin{tabular}{lcclc}
\hline Date & $\mu$ & $\left\langle v^{2}\right\rangle^{1 / 2}$ & $\left\langle\beta^{2}\right\rangle^{1 / 2}$ & $C_{\beta \delta_{v}}$ \\
\hline & & & & \\
10 Aug. 73 & 0.88 & 0.38 & 0.036 & +0.02 \\
28 Aug. 73 & 0.57 & 0.43 & 0.032 & -0.02 \\
Leighton et al. & - & 0.43 & 0.048 & +0.50 \\
\hline
\end{tabular}

with values obtained by Leighton et al. (1962) for the same line. There is in the present data an absence of brightness-velocity correlation at $60 \mathrm{~m} \AA$ from the line core and an overall decrease of the rms brightness fluctuation as compared with the earlier results $100 \mathrm{~m} \AA$ from the line core. In Table II, the second column indicates the disk position $(\mu=\cos \theta)$ of the observations.

\subsection{TWO-DIMENSIONAL AUTOCORRELATION FUNCTIONS}

Small squares in the velocity pictures were autocorrelated in order to reconnoiter 
the question of size and separation of velocity cells. This information resides quantitatively in the widths of autocorrelation peaks and in the positions of secondary peaks in the autocorrelation (AC) functions.

Six small 65 -element $\times 65$-element areas $\left(27 \times 27^{\prime \prime}\right)$ were investigated: three in each
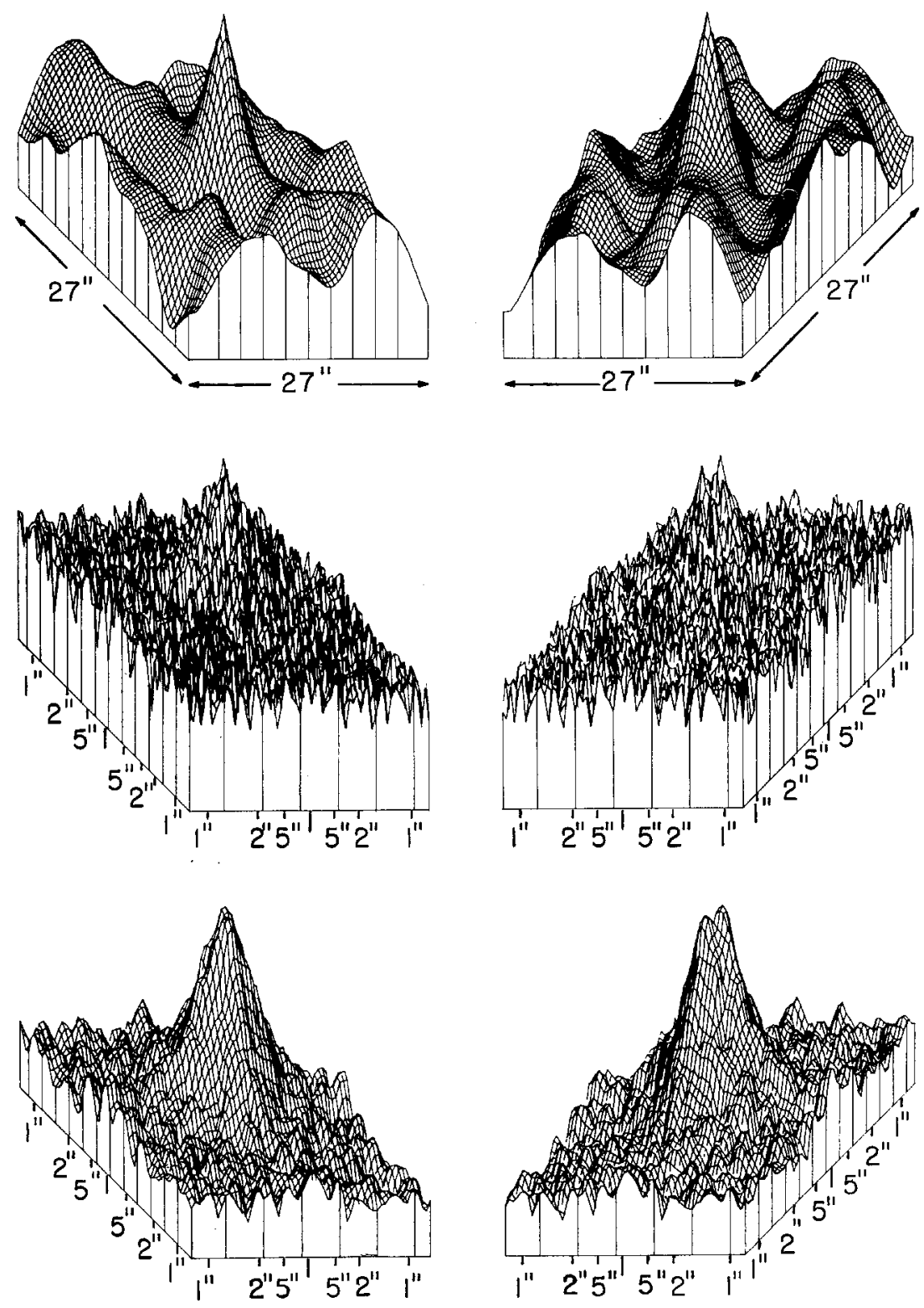

Fig. 1. AC function (upper), logarithm of modified power spectral density (middle) and logarithm of smoothed power spectral density (lower) for a $27 \times 27^{\prime \prime}$ square from the 10 August 1973 data. Two oblique views of each function are shown. Data points are separated by $0.42^{\prime \prime}$. Wavelength scales on the power spectra are marked in arcsec. 
of the two velocity pictures. The positions on the photosphere of three of these areas may be seen in Figure 1 of Teske (1974b). Because of computing time limitations an anaiysis of larger areas could not easily be accomplished using the $0.42^{\prime \prime}$ resolution. Hence the velocity data were also combined using 3-element $\times 3$-element
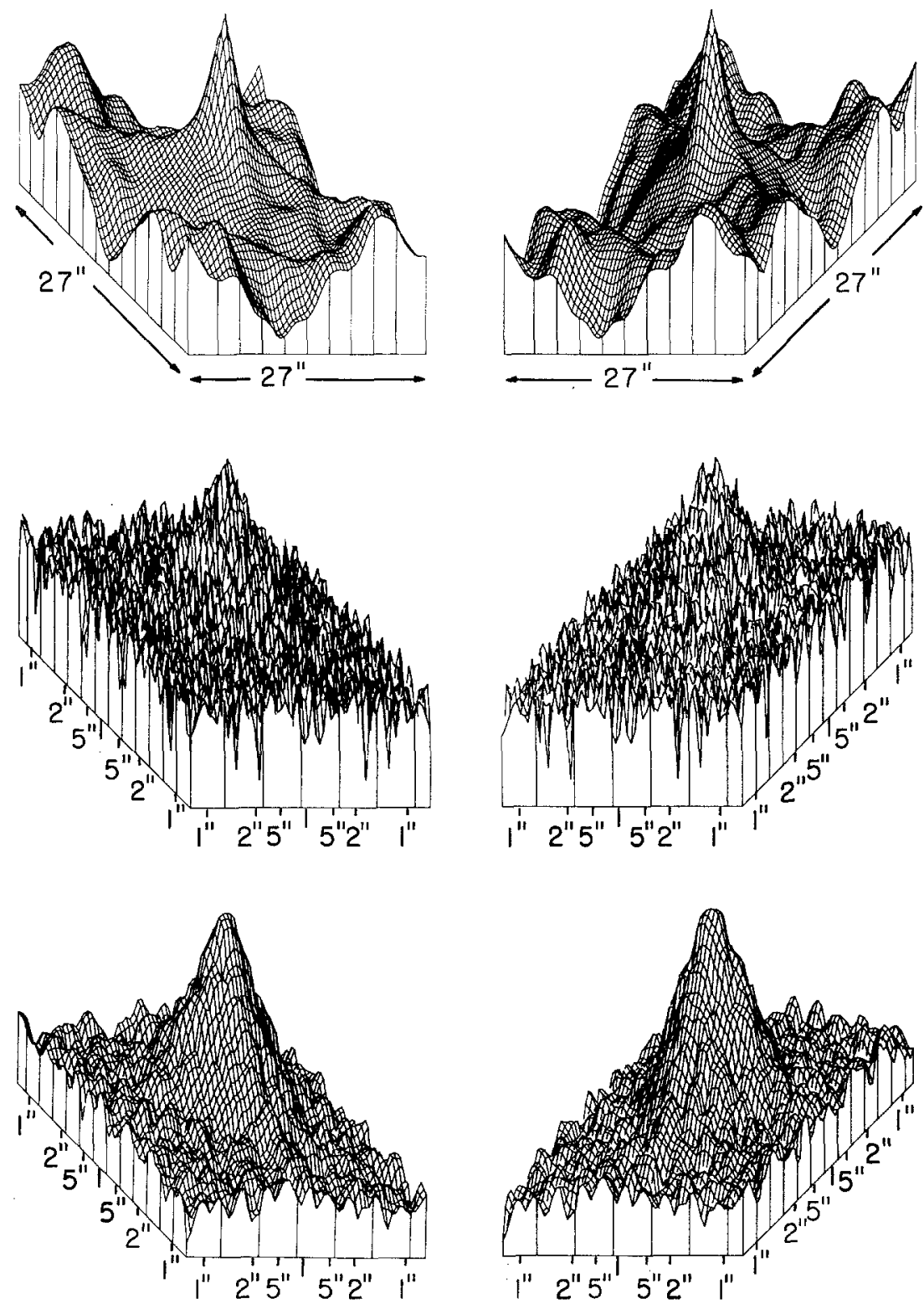

Fig. 2. AC function (upper), logarithm of modified power spectral density (middle) and logarithm of smoothed power spectral density (lower) for a $27 \times 27^{\prime \prime}$ square from the 28 August 1973 data. Two oblique views of each function are shown. Data points are separated by $0.42^{\prime \prime}$. Wavelength scales on the power spectra are marked in arcsec. 
averages to give an effective resolution of $1.25^{\prime \prime}\left(\lambda_{\text {Nyquist }}=1840 \mathrm{~km}\right.$ on the Sun). At this resolution, four larger areas were also analyzed: one $81 \times 81^{\prime \prime}$ square and one $125 \times 125^{\prime \prime}$ square in each of the two velocity pictures. These larger areas were approximately centered on the positions of the smalier $\left(27 \times 27^{\prime \prime}\right)$ areas.

Some of the two-dimensional AC functions for these areas are shown in Figures 1, 2 and 3. Two oblique views of each function are presented. The $\mathrm{AC}$ functions are reflectively symmetric about the central peak in the sense that rotation of the front two quadrants by $180^{\circ}$ about the central peak as axis yields the rear two quadrants. Diagonal ridges and troughs are quite marked in Figure 2 and are evident in Figure 1. The prominence and orientation of these ridges and troughs is predictable by looking at the velocity distribution in each small area that was analysed. There, the velocity elements (as weli as clumps or aggregations of them) are sometimes locally elongated and lie at angles to one another such as to produce the observed structure in the AC functions [see Figure 1 of Teske (1974b)].

These locally-elongated structures may be simply the supergranular boundaries. If that were so, the direction to the center of the disk would tend to lie nearly per-
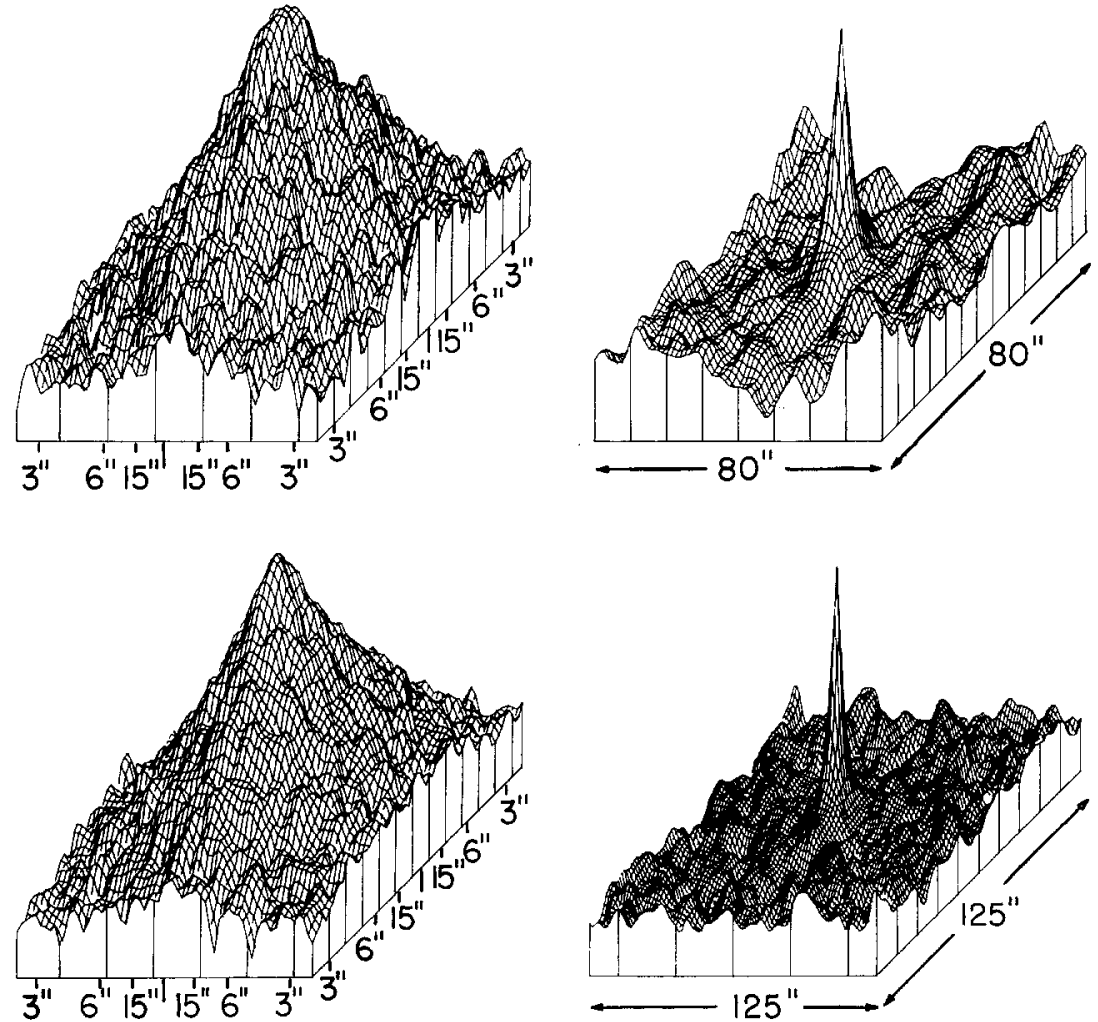

Fig. 3. AC functions and logarithmic smoothed power spectral density for an $81 \times 81^{\prime \prime}$ square (upper) and $125 \times 125^{\prime \prime}$ square (lower) from the 28 August 1973 data. Only one view of each function is shown. Data points are separated by $1.25^{\prime \prime}$. Wavelength scales on the power spectra are marked in aresec. 
pendicular to the ridges and troughs. Actualiy, in Figures 1 and 2 the local radius to the disk center lies nearly along them. In most of the AC functions which show elongated structures the local solar radius lies at shallow angles to the elongations. Thus the AC functions portray non-circularly symmetric velocity structures which are not directly associated with the network boundaries.

These elongated features might be produced by horizontal propagation of waves during the spectroheliograph scan. For this to be so, apparent horizontal speeds of 1500 to $2000 \mathrm{~km} \mathrm{~s}^{-1}$ would be required.

The evident lack of azimuthal symmetry in the AC functions calls into question the assumption of circular symmetry which is often invoked by observers (e.g., Beckers and Parnell, 1968; Frazier, 1968; Mehltretter, 1971) in manipulating bidimensional transforms and data, and is one of the problems to be discussed in the next section.

For all six of the $27 \times 27^{\prime \prime}$ squares the mean full width at half maximum (FWHM) in the AC peaks was 2480 ( $\pm 240 \mathrm{rms}$ ) km, or $3.4^{\prime \prime}$, along the length of the spectroheliograph entrance slit. The mean FWHM in the direction perpendicular to the entrance slit - that is, along the direction in which the slit was scanned - was 3050 $( \pm 640 \mathrm{rms}) \mathrm{km}$, attesting to the broadening effects of the one arcsec slit width. The mean ratio

$$
\frac{(\mathrm{FWHM})_{\perp}}{(\mathrm{FWHM})_{\|}}=1.23 \pm 0.22
$$

is close to the theoretical value of 1.17 for circular structures of size $2500 \mathrm{~km}$ on the Sun. In the individual AC functions, however, the ratio of FWHM taken perpendicular to the slit and parallel to it ranged from 1.00 to 1.67. This, together with the apparent ridges and troughs in the AC functions, indicates that the velocity 'cells' are not circularly symmetric nor uniformly placed geometrically.

For the four larger squares of lower effective resolution the mean FWHM parallel to the scanning slit is 3740 ( $\pm 240 \mathrm{rms}$ ) $\mathrm{km}$, or $4.7^{\prime \prime}$. Examples of the AC functions for these larger data samples in Figure 3 tend to have less strong asymmetries the ridges and troughs are less clearly seen - than is the case for the smaller data samples. Further, the range in the FWHM ratio for the central peaks is only 1.03 to 1.19 for the four cases. The larger data samples, which include many more velocity elements than do the small samples, therefore tend to portray them as more symmetric. Statistical fluctuations in the velocity cell structure which produced the asymmetries in the $\mathrm{AC}$ functions of small data samples are less severe when larger data samples are employed.

Secondary peaks are seen in the two dimensional AC functions. Simon and Leighton (1964), in their investigation of the supergranulation, showed that the locations of secondary peaks provided information about the spacing of supergranular elements and ultimately also specified their diameters because they are tightly packed. The amplitudes and positions of secondary peaks in the present data can be fitted 
by a model in which the velocity elements have a random distribution, as we now show.

The positive secondary peaks in the two-dimensional $\mathrm{AC}$ functions computed here were examined in the six small squares, in both $81 \times 81^{\prime \prime}$ squares and in one $125 \times 125^{\prime \prime}$ square. Figure 4 shows the distribution of distances of secondary AC peaks from the central peak. No regular spacing for the velocity elements can be seen. In the $27 \times 27^{\prime \prime}$ squares, the number of secondary peaks varied from three (two cases) to none (one case). Table III lists the counted numbers of secondary peaks per AC

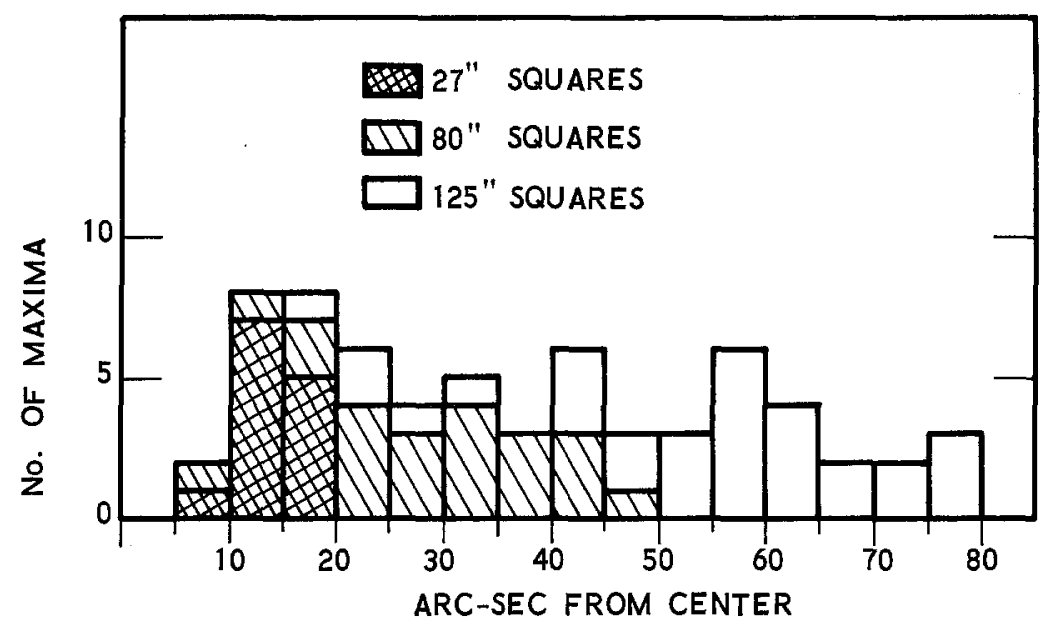

Fig. 4. The distances of positive secondary peaks from the central peak in the two dimensional AC functions show only a random spacing for velocity elements.

TABLE III

Numbers and amplitudes of secondary peaks in AC functions

\begin{tabular}{lllll}
\hline $\begin{array}{l}\text { Square } \\
\text { size }\end{array}$ & $\begin{array}{l}\text { Observed } \\
\text { number } \\
\text { of peaks } \\
\text { per square }\end{array}$ & $\begin{array}{l}\text { Observed } \\
\text { mean ampl. } \\
\text { of peaks }\end{array}$ & $\begin{array}{l}\text { Predicted } \\
\text { number } \\
\text { of peaks } \\
\text { per square }\end{array}$ & $\begin{array}{l}\text { Predicted } \\
\text { mean ampl. } \\
\text { of peaks }\end{array}$ \\
\hline $27 \times 27^{\prime \prime}$ & 1.5 & 0.25 & 1.4 & 0.30 \\
$81 \times 81^{\prime \prime}$ & 11.0 & 0.10 & 12.4 & 0.10 \\
$125 \times 125^{\prime \prime}$ & 30 & 0.065 & 30.0 & 0.065 \\
\hline
\end{tabular}

square and their mean amplitude (columns 2 and 3). The counted numbers of secondary $\mathrm{AC}$ peaks suggest that within a square $23 \times 23^{\prime \prime}$ we should on the average find just two velocity elements and hence one secondary AC peak. The number of randomly-distributed velocity elements will grow as the area being surveyed grows, so that we can predict on basis of area ratios the numbers of secondary AC peaks which should be seen, and these are given in column 4 of Table III. These are in good agreement with the numbers actually counted. 
If the velocity elements are randomly distributed on the photosphere, the amplitudes of secondary peaks should decline inversely as the square root of the area being surveyed. On this basis we can predict the mean amplitudes of the secondary peaks that are listed in column 5 of Table III. Those numbers are normalized to the mean amplitude for the $125 \times 125^{\prime \prime}$ square. Good agreement can also be obtained by normalizing to mean amplitudes for either of the other kinds of square. The requirement for normalization indicates that all velocity cells in the data are not alike in their velocity amplitude, a trivial statement.

The AC functions thus imply that the velocity cells are more or less randomly distributed on the photosphere. The cells are roughly of size 4 to $5^{\prime \prime}$. The numbers of secondary AC peaks per unit area and the observed mean amplitudes of the peaks may be fitted with a model in which the cells are randomly distributed but, in the mean, they seem to be spaced 10 to $15^{\prime \prime}$ apart. These results are in reasonable agreement with Deubner (1969), who obtained a two dimensional velocity map of the photospheric oscillations with a resolution of $\approx 3^{\prime \prime}$. His map shows that velocity elements of the same sign tend to be spaced 10 to $15^{\prime \prime}$ apart with a mean cell size in the range 7 to $8^{\prime \prime}$.

The FWHM of the AC functions can be used to specify a mean cell size for velocity elements. However an examination of the details of the AC functions shows that the cells are neither circularly symmetric nor uniformly distributed (spatially periodic). For this reason they may be considerably 'blurred' in power spectral densities.

\subsection{TWO-DIMENSIONAL POWER SPECTRA}

Two-dimensional power spectral densities were obtained from 64-element $\times$ 64-element Fourier transforms of the two dimensional AC functions. The AC functions were apodized prior to taking transforms (Brault and White, 1971) and the transforms smoothed by taking means (Edmonds and Webb, 1972a). These power spectra were plotted with a three dimensional computer plotting routine which displayed $\log P\left(k_{x}\right.$, $\left.k_{y}\right)$ on the $\left(k_{x}, k_{y}\right)$ plane.

Figure 5 shows the two-dimensional velocity power spectral distribution which results when an $\mathrm{AC}$ function is not apodized prior to transforming it. Brault and White (1971) have discussed the apodization problem. Their recommendations were followed. In the work reported here a cosine bell data window was adopted that affected the seven data points closest to each edge of the two-dimensional AC function.

Figures 1 and 2 show the $\mathrm{AC}$ functions and their transforms, as $\log P\left(k_{x}, k_{y}\right)$, for two of the $27 \times 27^{\prime \prime}$ squares. Some small residuum of the crossarms that are seen in Figure 5 remains in these transforms. The FFT power spectra are quite noisy because of the statistical incompleteness of the small data samples. Edmonds and Webb (1972a) have examined the problem of smoothing such transforms and have concluded that smoothing by averaging is useful although not advantageous from the standpoint of computing time. Accordingly, a running $3 \times 3$ mean has been taken of the raw transforms. The resulting two-dimensional power spectra (seen in two views at the bottom of Figures 1 and 2) are thus modified by the dual processes of 


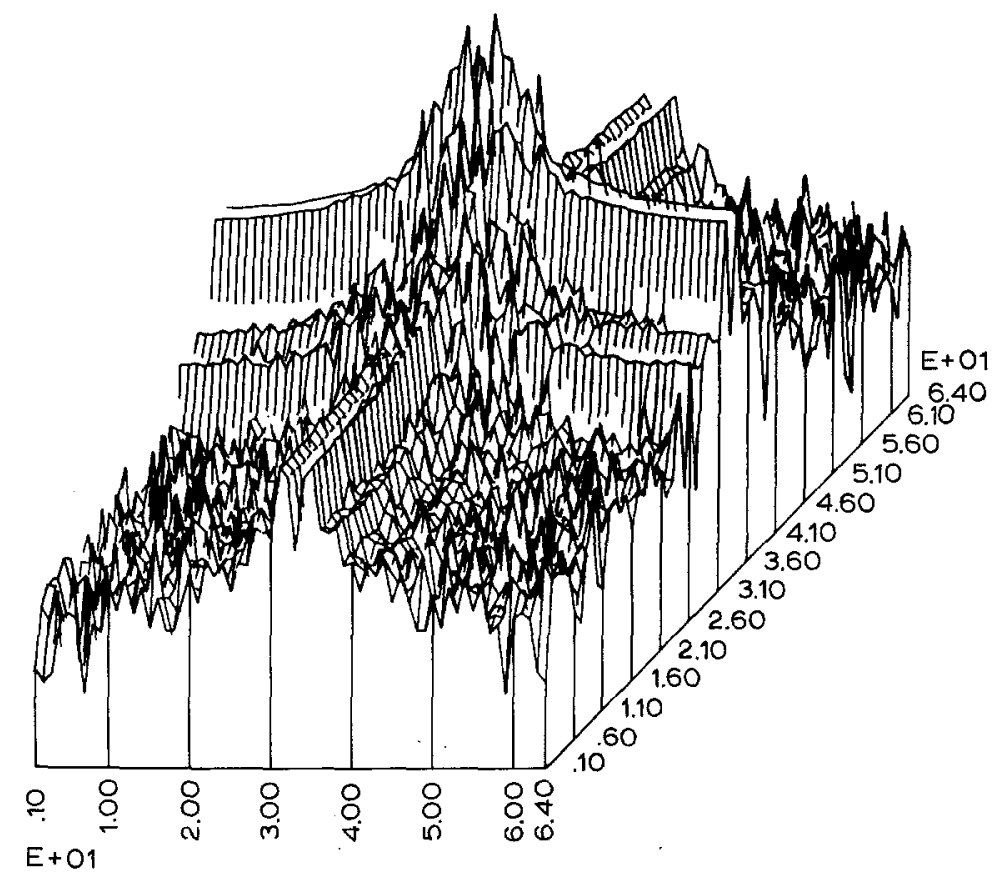

Fig. 5. An un-modified two-dimensional power spectrum which was derived from a non-apodized AC function.

apodization and smoothing. Experimentation with these processes has indicated that no major spatial information has been lost. Figure 3 shows AC functions and modified two-dimensional power spectral densities for a pair of the co-centered $81 \times 81^{\prime \prime}$ and $125 \times 125^{\prime \prime}$ squares.

The lack of azimuthal symmetry in the $\mathrm{AC}$ functions for $27 \times 27^{\prime \prime}$ squares is marked. However in the larger data samples this lack becomes less apparent because of the approach to statistical completeness in the data. There is a corresponding lack of azimuthal symmetry in the two-dimensional power spectra for the small squares which becomes less apparent in the power spectra of the larger squares for the same reason. The symmetry of the two-dimensional power spectra may be examined by inspecting the power distribution along their principal diagonals. Figure 6 shows the power spectral density along the principal diagonals in the modified power spectra of two of the smail squares. From an inspection of power spectra for all six small squares we find (i) that there are no stable resolved spectral features and (ii) that while the transient details of $\log P$ along different directions in the $\left(k_{x}, k_{y}\right)$ plane are different (cf. Figure 6), the gross power spectral distributions have the same slopes roughly independent of azimuth. That is, although statistical fiuctuations of velocity distribu- 


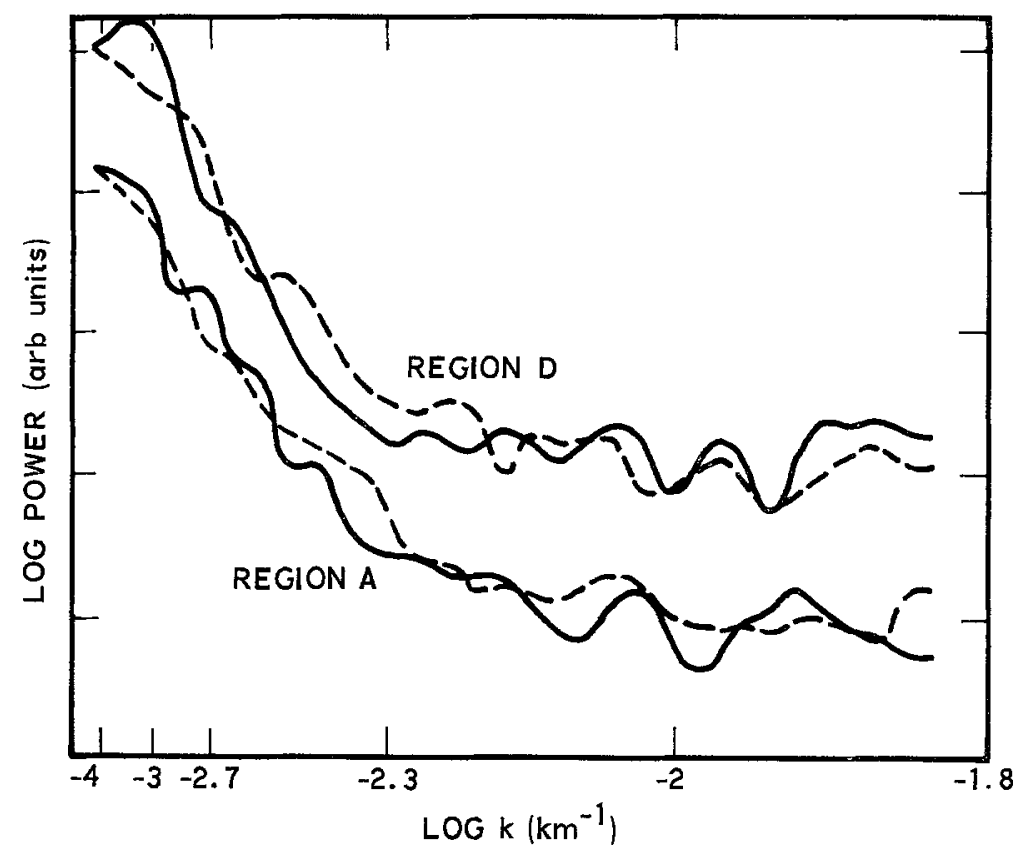

Fig. 6. Power spectral densities along the two principal diagonals of the power spectral density distributions shown in Figure 1 ('Region A') and in Figure 2 ('Region D').

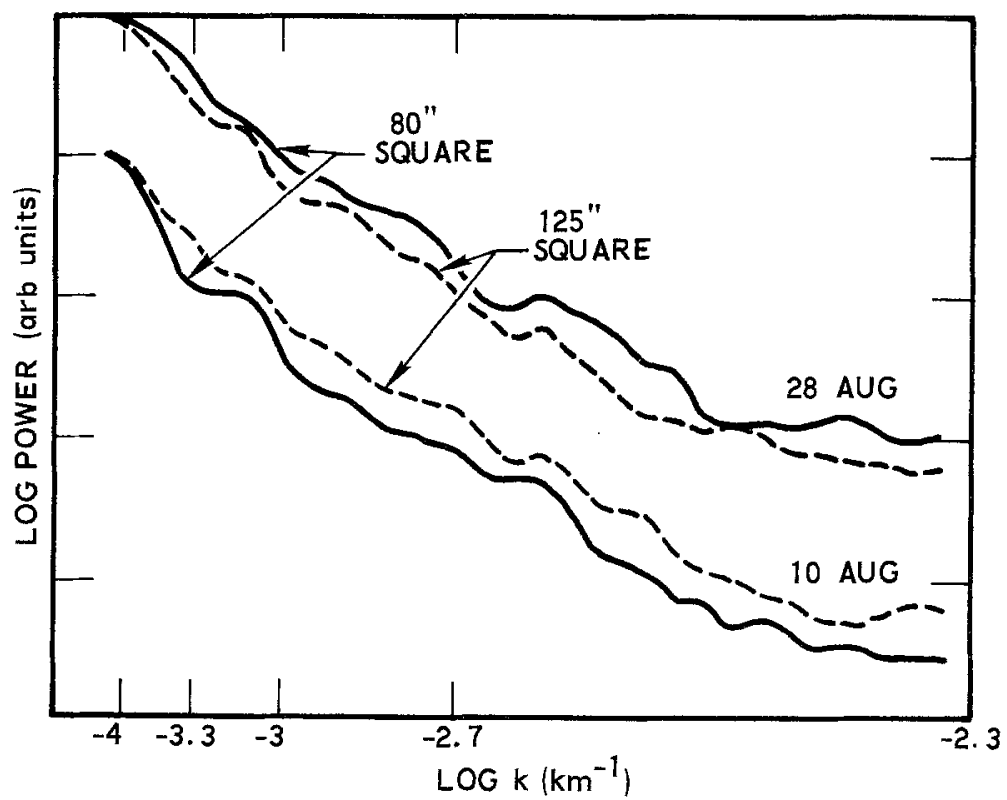

Fig. 7. Means of the power spectral densities along principal diagonals for the larger data samples. 
tions in small data samples produce spurious, unstable peaks in power spectra, the general trend of the gross power distribution seems correctly revealed. The principal diagonals of power spectra of the larger squares are more similar to one another than in the case of the small squares. Accordingly the assumption of azimuthal symmetry is better warranted for power spectral densities of large data samples. The means of the principal diagonals for the larger data samples are displayed in Figure 7. It should be obvious that taking averages of individual power spectra is equivalent to averaging $\mathrm{AC}$ functions prior to taking the transform of the average $\mathrm{AC}$ function.

Returning to the small squares: the power spectral densities for these do not resolve well any velocity structures larger than cell sizes $\sim 2000 \mathrm{~km}(\log k \lesssim-2.8)$. There are weak unstable features in the range of cell sizes $\sim 800$ to $1500 \mathrm{~km}$ although the smaller-sized features are not reliably portrayed because of seeing.

In the mean power spectral densities for the larger data samples (cf. Figure 7)

for which $\log k_{\text {Nyquist }} \sim-2.5$, stable resolved features at $\log k \sim-3.1$ and $\log k \sim-2.6$ appear. These may represent the velocity cells (here seen at a size $\sim 3900 \mathrm{~km}$ or $5.3^{\prime \prime}$ ) and vestigial, badly-smeared granules (here seen at a size $\sim 1250 \mathrm{~km}$ or $1.7^{\prime \prime}$ ). These latter are probably not resolved even in the $0.42^{\prime \prime}$ data. The spectral features are however broad and not prominent, and they do not extend beyond the $95 \%$ confidence interval as calculated from the variance of the mean.

Tannenbaum et al. (1969) found a power peak in the $(k, \omega)$ plane lying at $\log k \approx$ -3.6 and ascribed the low wavenumber of the peak to accidentally-coherent oscillations of randomly-placed cells of much smaller dimensions. The present power spectra have essentially no resolution at that wavenumber and cannot be used to verify the existence of a peak at $\log k \approx-3.6$. They strongly indicate, however, that the velocity elements of 'classical' size have enough randomness in separations and possibly in sizes as to inhibit their portrayal as easily-seen features in two dimensional spatial power spectra.

\section{Conclusions}

Two dimensional autocorrelation functions of photospheric velocities observed in the $\mathrm{Ca}$ I $\lambda 6103$ line have central peaks whose widths strongly suggest the presence of velocity elements of size about $5^{\prime \prime}$.

Two dimensional power spectral density distributions of the velocity fluctuations do not display strong power concentrations at wavenumbers appropriate to that 'classical' size of oscillatory elements. Were these cells indeed characterized by a fairly uniform $k_{h}=\left(k_{x}^{2}+k_{y}^{2}\right)^{1 / 2}$ and were they spatially periodic, they should be clearly seen in the two dimensional power spectra, whatever the values of $k_{x}$ and $k_{y}$ for each cell, as a circularly-symmetric ridge. Because the expected ridge is absent, we infer that the velocity cells tend to be spatially aperiodic; variations in their size and randomness in their distribution on the photosphere has blurred their appearance in power spectra derived from velocity snapshots of the sort used here.

The departure from azimuthal symmetry of two dimensional AC functions and their two dimensional Fourier transforms is not strong, and the departure decreases 
as the data sample area increases. Departures from azimuthal symmetry are entirely a matter of data sample size. Thus one dimensional techniques are useful and proper so long as the data sample is large.

Finally it appears that the failure of one dimensional sampling techniques to portray the spatial power peak for 'classical' 5-min oscillations is not due to the use of one dimensional analysis. Rather it is perhaps inherent in the use of power spectrum techniques to characterize a quasi-random distribution of velocity cells.

\section{Acknowledgements}

It is a pleasure to thank Drs E. B. Mayfield and G. A. Paulikas for arranging for me to study at the Aerospace Corp. Dr G. A. Chapman has been the source of many stimulating conversations and ideas. Thanks are due to J. Frawley for use of her computer programs and for much help, and to Dr P. Richter of California State University at Northridge for his two dimensional FFT program.

This research was supported by company funds of the Aerospace Corporation.

\section{References}

Apenzeller, J. and Schröter, E. H.: 1968, Solar Phys. 4, 131.

Beckers, J. M. and Parnell, R. L.: 1969, Solar Phys. 9, 39.

Brault, J. W. and White, O. R.: 1971, Astron. Astrophys. 13, 169.

Deubner, F. L.: 1967, Solar Phys. 2, 133.

Deubner, F. L.: 1969, Solar Phys. 9, 343.

Deubner, F. L.: 1972, Solar Phys. 22, 263.

Edmonds, F. N., Jr. and Webb, C. J.: 1972a, Solar Phys. 22, 276.

Edmonds, F. N., Jr. and Webb, C. J.: 1972b, Solar Phys. 25, 44.

Evans, J. W. and Michard, R.: 1962, Astrophys. J. 136, 493.

Frazier, E. N.: 1968, Z. Astrophys. 68, 345.

Leighton, R. B., Noyes, R. W., and Simon, G. W.: 1962, Astrophys. J. 135, 474.

Mayfield, E. B.: 1971, in R. Howard (ed.), 'Solar Magnetic Fields', IAU Symp. 43, 376.

Mehltretter, J. P.: 1971, Solar Phys. 16, 253.

Mein, P.: 1966, Ann. Astrophys. 29, 153.

Reif, R. J. and Musman, S.: 1971, Solar Phys. 20, 257.

Reiling, H.: 1971, Solar Phys. 19, 297.

Roddier, F.: 1966, Ann. Astrophys. 29, 639.

Sheeley, N. R., Jr. and Bhatnagar, A.: 1971, Solar Phys. 18, 379.

Simon, G. W. and Leighton, R. B.: 1964, Astrophys. J. 140, 1120.

Tannenbaum, A. S., Wilcox, J. M., Frazier, E. N., and Howard, R.: 1969, Solar Phys. 9, 328.

Teske, R. G.: 1974a, Aerospace Technical Report ATR-75(8102)-1.

Teske, R. G.: 1974b, to be submitted to Solar Physics.

Wolf, C.: 1973, Solar Phys. 32, 31. 